\title{
Effect of Integrated Nitrogen Management and Spacing on Yield, Quality and Economics of Fodder Maize (Zea mays L.) Var. Shiats Makka-2
}

\author{
Choudary Pradeep Kumar ${ }^{1}$, Rajesh Singh ${ }^{1 *}$ and A.C. Singh ${ }^{2}$ \\ ${ }^{1}$ Department of Agronomy, SHUATS, Allahabad, India \\ ${ }^{2}$ Department of Agronomy, KAPG College, Allahabad State University, Allahabad, India \\ *Corresponding author
}

\section{A B S T R A C T}

\section{Key words \\ Integrated nitrogen management, Vermicompost, \\ Azotobacter, Spacing, \\ Green fodder yield, \\ Quality and economics \\ Article Info \\ Accepted: \\ 10 September 2018 \\ Available Online: \\ 10 October 2018}

\begin{abstract}
A field experiment was conducted during the rabi season of 2017-18 on fodder maize crop (var. SHIATS Makka- 2) at the Crop Research Farm, Department of Agronomy, Naini Agricultural Institute, SHUATS, Allahabad (U.P.). The soil of the experimental field was sandy loam with low organic carbon $(0.45 \%)$ and a soil $\mathrm{pH}$ of 7.6. The experiment comprised of three planting geometry viz., $40 \times 10 \mathrm{~cm}, 50 \times 10 \mathrm{~cm}, 60 \times 10 \mathrm{~cm}$ and 2 nitrogen levels $90 \mathrm{~kg} \mathrm{~N} \mathrm{ha}^{-1}$ and $120 \mathrm{~kg} \mathrm{~N} \mathrm{ha}^{-1}$ respectively, with 18 treatments replicated thrice and laid out in Randomized Block Design. The experiment was conducted to evaluate the growth and yield of fodder maize (Zea mays L.). The result revealed that treatment $\mathrm{T}_{17}\left[120 \mathrm{~kg} \mathrm{~N}^{-1}+(50 \% \mathrm{~N}\right.$ through vermicompost $+50 \% \mathrm{~N}$ through urea $)+$ Seed inoculated with Azotobacter $+50 \times 10 \mathrm{~cm}$ ] recorded higher crude protein (9.567), Ash (5.270\%), Gross return (₹ 89120 ha $\left.^{-1}\right)$, Net Return (₹ 51351.6 ha $\left.^{-1}\right)$, B: C (2.36) ratio.
\end{abstract}

\section{Introduction}

Maize (Zea mays L.) is one of the most versatile and multi utility cereals and commonly known as queen of cereals due to its highest genetic yield potential and wider adaptability under diverse agro-ecological conditions. In India area under fodder crops is only $8.4 \mathrm{~m}$ ha which is static since last two decades.

At present, the country faces a net deficit of $61.1 \%$ green fodder, $21.9 \%$ dry crop residue and 64\% feeds (Chaudhary et al., 2012). Among cultivated forage crops, maize is most suitable crop for fodder as well as silage because of its high yielding ability and excellent nutritional profile. Besides, it is served as source of food, feed and industrial raw material and provides enormous opportunity for crop diversification, value addition and employment generation. Worldwide, maize is an ideal fodder crop because of its quick growing nature, succulence, palatability and excellent quality without any anti-nutritional factor; when harvested at any stage of crop growth (Anonymous, 2013). Forage maize responds differently to plant densities under different environmental and cultural factors, which influence maize forage yield and quality (Carpici et al., 2010). 
Furthermore, the fertilizer management is one of the most important factors that influence the growth and yield of maize crop. In fact, organic manure not only provides plant nutrients but also improves or sustains the soil health. The micronutrient content in organic manure may be sufficient enough to meet the crop production requirement but problem of low soil fertility is one of the obstacles to maintain and sustain agricultural production and productivity. Integrated nutrient management (INM) is a judicious use of organic and inorganic sources of nutrients to crop field (Kannan et al., 2013).

Nitrogen $(\mathrm{N})$ sources and rates influence grain yield and profitability and total dry matter accumulation, agronomic $\mathrm{N}$-use efficiency and harvest index in maize (Zea mays L.). Planting geometry directly influences the plant population $/ \mathrm{m}^{2}$ or the fodder production $/ \mathrm{m}^{2}$. Keeping this in view, the present experiment was undertaken to study the effect of planting geometry and integrated Nitrogen on quality and yield of fodder maize.

\section{Materials and Methods}

A field experiment was conducted during the rabi season of 2017 on fodder maize crop (var. SHIATS Makka-2) at Crop Research Farm, Department of Agronomy, Naini Agricultural Institute, SHUATS, Allahabad (U.P.), which is located at $25^{\circ} 24^{\prime} 41.27^{\prime \prime} \mathrm{N}$ latitude, $81^{\circ}$ 51'3.42" E longitude and at an altitude of 98 $\mathrm{m}$ above the mean sea level. The soil of experimental field was sandy loam having $\mathrm{pH}$ of 7.2 with $0.45 \%$ organic carbon. The experiment consisted of 18 treatments with two levels of nitrogen i.e., levels $90 \mathrm{~kg} \mathrm{~N}^{-1}$ and $120 \mathrm{~kg} \mathrm{~N} \mathrm{ha}^{-1}$ respectively, managed through three different sources viz. $50 \% \mathrm{~N}$ through vermicompost $+50 \% \mathrm{~N}$ through urea, $50 \% \mathrm{~N}$ through vermicompost $+50 \% \mathrm{~N}$ through urea in addition with seed inoculation with Azotobacter, which was compared with
$100 \% \mathrm{~N}$ supplied through urea alone with three different row spacing i.e. $40 \mathrm{~cm}, 50 \mathrm{~cm}$ and $60 \mathrm{~cm}$ at a plant to plant spacing of $10 \mathrm{~cm}$, laid out in Randomized Block Design and replicated thrice. Fodder maize (var. SHIATS Makka-2) was sown $6^{\text {th }}$ of November 2017. The crop matured in 90 days and was harvested in first week of February. After harvesting, the data on yield attributes viz. green fodder yield $\left(\mathrm{t}^{-1}\right)$, dry weight $(\mathrm{g})$ and plant height $(\mathrm{cm})$ are statistically analyzed and critical difference were calculated.

\section{Results and Discussions}

\section{Green fodder yield}

The data pertaining to green fodder yield and quality are presented in Table 1, which revealed that the integrated nitrogen management in fodder maize (var. SHIATS Makka-2), significantly increase the green fodder yield (44.56 $\mathrm{t} \mathrm{ha}^{-1}$ ) was observed statistically significant with treatment $\mathrm{T}_{17}(120$ $\mathrm{kg} \mathrm{N} \mathrm{ha}{ }^{-1}+50 \% \mathrm{~N}$ through $\mathrm{VC}+50 \% \mathrm{~N}$ through Urea + Seed inoculation with Azotobacter $+50 \mathrm{~cm} \times 10 \mathrm{~cm}$ ), are significantly higher with $\mathrm{T}_{17}$ This result are very close with the finding of Arif et al., (2010) and Barik et al., (2007).

\section{Effect on quality}

As given in Table 2, the quality vaz. The maximum Crude protein $(9.567 \%)$ and Ash $(5.720 \%)$ were found in treatment $\mathrm{T}_{17}(120 \mathrm{~kg}$ $\mathrm{N} \mathrm{ha}{ }^{-1}+50 \% \mathrm{~N}$ through $\mathrm{VC}+50 \% \mathrm{~N}$ through Urea + Seed inoculation with Azotobacter + $50 \times 10 \mathrm{~cm}$ ). The results are in accordance with Joshi and Kumar (2007) who also reported significant increase in crude protein yield of maize with increase in levels of nitrogen up to $120 \mathrm{~kg} \mathrm{ha}{ }^{-1}$. And the lower crude fiber $(23.853 \%)$ was found under treatment $\mathrm{T}_{6}(90$ $\mathrm{kg} \mathrm{N} \mathrm{ha}{ }^{-1}+50 \% \mathrm{~N}$ through $\mathrm{VC}+50 \% \mathrm{~N}$ through Urea $+60 \times 10 \mathrm{~cm}$ ). 
Table.1 Effect of integrated nitrogen management and spacing on yield and quality of fodder maize var. Shiats Makka-2

\begin{tabular}{|c|c|c|c|c|c|}
\hline \multicolumn{2}{|r|}{ Treatments } & \multicolumn{3}{|c|}{ Proximate Analysis } & \multirow{2}{*}{$\begin{array}{c}\text { Green } \\
\text { fodder } \\
\text { yield } \\
\left(\mathrm{t} \mathrm{ha}^{-1}\right)\end{array}$} \\
\hline & & $\begin{array}{c}\text { Crude } \\
\text { protein } \\
(\%)\end{array}$ & $\begin{array}{c}\text { Crude } \\
\text { fibre }(\%)\end{array}$ & Ash (\%) & \\
\hline T1 & $90 \mathrm{~kg} \mathrm{~N} \mathrm{ha}^{-1}+100 \% \mathrm{~N}$ through Urea $+40 \times 10 \mathrm{~cm}$ & 6.947 & 31.473 & 2.367 & 38.00 \\
\hline T2 & $90 \mathrm{~kg} \mathrm{~N} \mathrm{ha}^{-1}+100 \% \mathrm{~N}$ through Urea $+50 \times 10 \mathrm{~cm}$ & 7.537 & 31.920 & 2.427 & 34.00 \\
\hline T3 & $90 \mathrm{~kg} \mathrm{~N} \mathrm{ha}^{-1}+100 \% \mathrm{~N}$ through Urea $+60 \times 10 \mathrm{~cm}$ & 7.653 & 31.333 & 2.213 & 35.00 \\
\hline T4 & $90 \mathrm{~kg} \mathrm{~N} \mathrm{ha}^{-1}+50 \% \mathrm{~N}$ through $\mathrm{VC}+50 \% \mathrm{~N}$ through Urea $+40 \times 10 \mathrm{~cm}$ & 7.863 & 30.240 & 2.777 & 33.50 \\
\hline T5 & $90 \mathrm{~kg} \mathrm{~N} \mathrm{ha}^{-1}+50 \% \mathrm{~N}$ through $\mathrm{VC}+50 \% \mathrm{~N}$ through Urea $+50 \times 10 \mathrm{~cm}$ & 9.083 & 26.050 & 5.333 & 34.80 \\
\hline T6 & $90 \mathrm{~kg} \mathrm{~N} \mathrm{ha}^{-1}+50 \% \mathrm{~N}$ through $\mathrm{VC}+50 \% \mathrm{~N}$ through Urea $+60 \times 10 \mathrm{~cm}$ & 9.030 & 23.853 & 5.267 & 33.00 \\
\hline T7 & $90 \mathrm{~kg} \mathrm{~N} \mathrm{ha}^{-1}+50 \% \mathrm{~N}$ through $\mathrm{VC}+50 \% \mathrm{~N}$ through Urea + Azotobacter $(\mathrm{SI})+40 \times 10 \mathrm{~cm}$ & 7.983 & 26.400 & 3.933 & 37.50 \\
\hline T8 & $90 \mathrm{~kg} \mathrm{~N} \mathrm{ha}^{-1}+50 \% \mathrm{~N}$ through $\mathrm{VC}+50 \% \mathrm{~N}$ through Urea + Azotobacter $(\mathrm{SI})+50 \times 10 \mathrm{~cm}$ & 8.110 & 31.567 & 5.497 & 39.00 \\
\hline T9 & $90 \mathrm{~kg} \mathrm{~N} \mathrm{ha}^{-1}+50 \% \mathrm{~N}$ through $\mathrm{VC}+50 \% \mathrm{~N}$ through Urea + Azotobacter $(\mathrm{SI})+60 \times 10 \mathrm{~cm}$ & 9.177 & 33.233 & 5.360 & 35.50 \\
\hline T10 & $120 \mathrm{~kg} \mathrm{~N} \mathrm{ha}^{-1}+100 \% \mathrm{~N}$ through Urea $+40 \times 10 \mathrm{~cm}$ & 7.560 & 25.363 & 3.843 & 33.60 \\
\hline T11 & $120 \mathrm{~kg} \mathrm{~N} \mathrm{ha}^{-1}+100 \% \mathrm{~N}$ through Urea $+50 \times 10 \mathrm{~cm}$ & 8.033 & 26.143 & 4.233 & 40.00 \\
\hline T12 & $120 \mathrm{~kg} \mathrm{~N} \mathrm{ha}^{-1}+100 \% \mathrm{~N}$ through Urea $+60 \times 10 \mathrm{~cm}$ & 8.053 & 28.603 & 4.533 & 41.00 \\
\hline T13 & $120 \mathrm{~kg} \mathrm{~N} \mathrm{ha}^{-1}+50 \% \mathrm{~N}$ through $\mathrm{VC}+50 \% \mathrm{~N}$ through Urea $+40 \times 10 \mathrm{~cm}$ & 8.103 & 25.700 & 3.203 & 42.50 \\
\hline T14 & $120 \mathrm{~kg} \mathrm{~N} \mathrm{ha}^{-1}+50 \% \mathrm{~N}$ through $\mathrm{VC}+50 \% \mathrm{~N}$ through Urea $+50 \times 10 \mathrm{~cm}$ & 8.917 & 24.120 & 5.190 & 42.66 \\
\hline T15 & $120 \mathrm{~kg} \mathrm{~N} \mathrm{ha}^{-1}+50 \% \mathrm{~N}$ through $\mathrm{VC}+50 \% \mathrm{~N}$ through Urea $+60 \times 10 \mathrm{~cm}$ & 8.120 & 26.080 & 5.230 & 42.00 \\
\hline T16 & $120 \mathrm{~kg} \mathrm{~N} \mathrm{ha}^{-1}+50 \% \mathrm{~N}$ through $\mathrm{VC}+50 \% \mathrm{~N}$ through Urea + Azotobacter $(\mathrm{SI})+40 \times 10 \mathrm{~cm}$ & 9.307 & 25.817 & 5.107 & 43.60 \\
\hline T17 & $120 \mathrm{~kg} \mathrm{~N} \mathrm{ha}^{-1}+50 \% \mathrm{~N}$ through $\mathrm{VC}+50 \% \mathrm{~N}$ through Urea + Azotobacter $(\mathrm{SI})+50 \times 10 \mathrm{~cm}$ & 9.567 & 28.983 & 5.720 & 44.56 \\
\hline \multirow[t]{4}{*}{ T18 } & $120 \mathrm{~kg} \mathrm{~N} \mathrm{ha}^{-1}+50 \% \mathrm{~N}$ through $\mathrm{VC}+50 \% \mathrm{~N}$ through Urea + Azotobacter $(\mathrm{SI})+60 \times 10 \mathrm{~cm}$ & 9.363 & 26.400 & 5.643 & 44.00 \\
\hline & F- test & $\mathrm{S}$ & $\mathrm{S}$ & $\mathrm{S}$ & S \\
\hline & S. Ed. $( \pm)$ & 0.542 & 1.203 & 0.406 & 1.018 \\
\hline & C. D. $(P=0.05)$ & 1.118 & 2.483 & 0.838 & 2.102 \\
\hline
\end{tabular}


Table.2 Effect of integrated nitrogen management and spacing on economics of fodder maize var. Shiats Makka-2

\begin{tabular}{|c|c|c|c|c|c|}
\hline \multicolumn{2}{|r|}{ Treatments } & \multicolumn{4}{|c|}{ Economics } \\
\hline & & $\begin{array}{c}\text { Cost of } \\
\text { Cultivation }\end{array}$ & $\begin{array}{l}\text { Gross } \\
\text { return } \\
\left(₹ \text { ha }^{-1}\right)\end{array}$ & $\begin{array}{c}\text { Net } \\
\text { Return } \\
\left(₹ \mathbf{h a}^{-1}\right)\end{array}$ & B:C ratio \\
\hline T1 & $90 \mathrm{~kg} \mathrm{~N} \mathrm{ha}^{-1}+100 \% \mathrm{~N}$ through Urea $+40 \times 10 \mathrm{~cm}$ & 38,339 & 76000 & 37661.1 & 1.98 \\
\hline T2 & $90 \mathrm{~kg} \mathrm{~N} \mathrm{ha}^{-1}+100 \% \mathrm{~N}$ through Urea $+50 \times 10 \mathrm{~cm}$ & 38,339 & 68000 & 29661.1 & 1.77 \\
\hline T3 & $90 \mathrm{~kg} \mathrm{~N} \mathrm{ha}^{-1}+100 \% \mathrm{~N}$ through Urea $+60 \times 10 \mathrm{~cm}$ & 38,339 & 70000 & 31661.1 & 1.83 \\
\hline T4 & $90 \mathrm{~kg} \mathrm{~N} \mathrm{ha}^{-1}+50 \% \mathrm{~N}$ through $\mathrm{VC}+50 \% \mathrm{~N}$ through Urea $+40 \times 10 \mathrm{~cm}$ & 37,453 & 67000 & 29546.8 & 1.79 \\
\hline T5 & $90 \mathrm{~kg} \mathrm{~N} \mathrm{ha}^{-1}+50 \% \mathrm{~N}$ through $\mathrm{VC}+50 \% \mathrm{~N}$ through Urea $+50 \times 10 \mathrm{~cm}$ & 37,453 & 69600 & 32146.8 & 1.86 \\
\hline T6 & $90 \mathrm{~kg} \mathrm{~N} \mathrm{ha}^{-1}+50 \% \mathrm{~N}$ through $\mathrm{VC}+50 \% \mathrm{~N}$ through Urea $+60 \times 10 \mathrm{~cm}$ & 37,453 & 66000 & 28546.8 & 1.76 \\
\hline T7 & $90 \mathrm{~kg} \mathrm{~N} \mathrm{ha}{ }^{-1}+50 \% \mathrm{~N}$ through $\mathrm{VC}+50 \% \mathrm{~N}$ through Urea + Azotobacter $(\mathrm{SI})+40 \times 10 \mathrm{~cm}$ & 37,453 & 75000 & 37546.8 & 2.00 \\
\hline T8 & $90 \mathrm{~kg} \mathrm{~N} \mathrm{ha}^{-1}+50 \% \mathrm{~N}$ through $\mathrm{VC}+50 \% \mathrm{~N}$ through Urea + Azotobacter $(\mathrm{SI})+50 \times 10 \mathrm{~cm}$ & 37,453 & 78000 & 40546.8 & 2.08 \\
\hline T9 & $90 \mathrm{~kg} \mathrm{~N} \mathrm{ha}^{-1}+50 \% \mathrm{~N}$ through $\mathrm{VC}+50 \% \mathrm{~N}$ through Urea + Azotobacter $(\mathrm{SI})+60 \times 10 \mathrm{~cm}$ & 37,453 & 71000 & 33546.8 & 1.90 \\
\hline T10 & $120 \mathrm{~kg} \mathrm{~N} \mathrm{ha}^{-1}+100 \% \mathrm{~N}$ through Urea $+40 \times 10 \mathrm{~cm}$ & 38,949 & 67200 & 28250.6 & 1.73 \\
\hline T11 & $120 \mathrm{~kg} \mathrm{~N} \mathrm{ha}^{-1}+100 \% \mathrm{~N}$ through Urea $+50 \times 10 \mathrm{~cm}$ & 38,949 & 80000 & 41050.6 & 2.05 \\
\hline T12 & $120 \mathrm{~kg} \mathrm{~N} \mathrm{ha}^{-1}+100 \% \mathrm{~N}$ through Urea $+60 \times 10 \mathrm{~cm}$ & 38,949 & 82000 & 43050.6 & 2.11 \\
\hline T13 & $120 \mathrm{~kg} \mathrm{~N} \mathrm{ha}^{-1}+50 \% \mathrm{~N}$ through $\mathrm{VC}+50 \% \mathrm{~N}$ through Urea $+40 \times 10 \mathrm{~cm}$ & 37,768 & 85000 & 47231.6 & 2.25 \\
\hline T14 & $120 \mathrm{~kg} \mathrm{~N} \mathrm{ha}^{-1}+50 \% \mathrm{~N}$ through $\mathrm{VC}+50 \% \mathrm{~N}$ through Urea $+50 \times 10 \mathrm{~cm}$ & 37,768 & 85320 & 47551.6 & 2.26 \\
\hline T15 & $120 \mathrm{~kg} \mathrm{~N} \mathrm{ha}^{-1}+50 \% \mathrm{~N}$ through $\mathrm{VC}+50 \% \mathrm{~N}$ through Urea $+60 \times 10 \mathrm{~cm}$ & 37,768 & 84000 & 46231.6 & 2.22 \\
\hline T16 & $120 \mathrm{~kg} \mathrm{~N} \mathrm{ha}{ }^{-1}+50 \% \mathrm{~N}$ through $\mathrm{VC}+50 \% \mathrm{~N}$ through Urea + Azotobacter $(\mathrm{SI})+40 \times 10 \mathrm{~cm}$ & 37,768 & 87200 & 49431.6 & 2.31 \\
\hline T17 & $120 \mathrm{~kg} \mathrm{~N} \mathrm{ha}^{-1}+50 \% \mathrm{~N}$ through $\mathrm{VC}+50 \% \mathrm{~N}$ through Urea + Azotobacter $(\mathrm{SI})+50 \times 10 \mathrm{~cm}$ & 37,768 & 89120 & 51351.6 & 2.36 \\
\hline T18 & $120 \mathrm{~kg} \mathrm{~N} \mathrm{ha}^{-1}+50 \% \mathrm{~N}$ through $\mathrm{VC}+50 \% \mathrm{~N}$ through Urea + Azotobacter $(\mathrm{SI})+60 \times 10 \mathrm{~cm}$ & 37,768 & 88000 & 50231.6 & 2.33 \\
\hline
\end{tabular}


The higher nitrogen doses result in higher protein synthesis and lower soluble carbohydrates which could be responsible for lower crude fiber content of the fodder maize. The results are in conformity with the findings of Almodares et al., (2009), Sujata et al., 2008 and Krishna et al., 1998.

\section{Effect on economics}

The maximum gross return (89120 Rs. ha $\left.{ }^{-1}\right)$, net return (51351.6 Rs. ha ${ }^{-1}$ ) and benefit cost ratio (2.36) was recorded in treatment $\mathrm{T}_{17}(120 \mathrm{~kg} \mathrm{~N}$ $\mathrm{ha}^{-1}+50 \% \mathrm{~N}$ through $\mathrm{VC}+50 \% \mathrm{~N}$ through Urea + Seed inoculation with Azotobacter + $50 \times 10 \mathrm{~cm})$. The plant spacing of $50 \mathrm{~cm} \times 10 \mathrm{~cm}$ gave the maximum green fodder yield then the other spacing. These results are in conformity with those of Kunjir (2004).

On the basis of the above findings, it can be concluded that for obtaining higher green fodder yield, better fodder quality and benefit cost ratio, SHAITS Makka-2, should be fertilizer with $120 \mathrm{~kg} / \mathrm{ha}$ of nitrogen $(\mathrm{N})$ as $50 \%$ $\mathrm{N}$ through vermicompost and 50\% $\mathrm{N}$ through urea in addition to seed inoculation with Azotobacter and should be sown at a spacing of $50 \mathrm{~cm}$ (row to row) $\times 10 \mathrm{~cm}$ (plant to plant).

\section{References}

Almodares, C.A., Jafarinia, M. and Hadi, M.R. (2009). The Effects of Nitrogen Fertilizer on Chemical Compositions in Corn and Sweet Sorghum. American-Eurasian J. Agr. \& Env. Sci., 6(4): 441-446.

Anonymous, 2013. Vision-2050, Indian Grassland and Fodder Research Institute (Indian Council of Agricultural Research) Jhansi.

Arif, M., Amin, I., Jan, M.T., Munir, I., Nawab, K., Khan, N.U. and Marwat, K.B. (2010).
Effect of plant population and nitrogen levels and methods of application on ear characters and yield of maize. Pakistan Journal of Botany, 42 (3): 1959-1967.

Barik, Bindhani, Anita, K.C., Garnayak, L.M. and Mahapatra, P.K. (2007). Nitrogen management in baby corn (Zea mays). Indian Journal of Agronomy, 52 (2): 135138.

Carpici, E.B., Necmettin, C. and Gamze, B. 2010. Yield and quality of forage maize as influenced by plant density and nitrogen rate. Turkish Journal of Field Crops, 15(2): 128-32.

Chaudhary, D.P., Kumar, A.S., Sapna, Mandhania, Srivastava, P. and Kumar, R.S. 2012. Maize as fodder? an alternative approach, directorate of maize research, Pusa Campus, New Delhi -110 012, Technical Bull., pp. 32.

Kannan, R.L., Dhivya, M., Abinaya, D., Krishna, R.L. and Kumar, S.K. (2013). Effect of Integrated Nutrient Management on Soil Fertility and Productivity in Maize. Bulletin of Environment, Phamacology and Life Sciences, 2 (8): 61-67.

Krishna, S.V., Raikhelkar, S.V. and Sambasiva, R. (1998). Effect of planting pattern and nitrogen on fodder maize intercropped with cowpea. Indian J. Agron., 43(2): 237-240.

Kunjir SS. Effect of planting geometry nitrogen levels and micronutrients on the maize (Zea mays L.) as affected by different crop geometry and level of nitrogen application. Research Paper, 2004, 8(8).

Sujata, M.G., Lingaraju, B.S., Palled, Y.B. and Ashalatha, K.V. (2008). Importance of integrated nutrient management practices in maize under rainfed conditions. Karnataka J. Agr. Sci., 21(3): 334-338.

\section{How to cite this article:}

Choudary Pradeep Kumar, Rajesh Singh and Singh, A.C. 2018. Effect of Integrated Nitrogen Management and Spacing on Yield, Quality and Economics of Fodder Maize (Zea mays L.) Var. Shiats Makka-2. Int.J.Curr.Microbiol.App.Sci. 7(10): 1269-1273.

doi: https://doi.org/10.20546/ijcmas.2018.710.142 